\title{
An Annulus Optimal Threshold Method Based for Non-uniform Illumination Image Correction
}

\author{
Yujie $\mathrm{Li}^{*}$, Huimin Lu, Shiyuan Yang, Lifeng Zhang, Seiichi Serikawa \\ Department of Electrical Engineering and Electronics, Kyushu Institute of Technology, Japan \\ *Corresponding Author: yzyjli@boss.ecs.kyutech.ac.jp
}

\begin{abstract}
The objects in the video supervisory systems, such as underwater detecting, industrial endoscope or pipeline detecting, due to the large noise and low contrast and scattering of light, we cannot directly use the classical threshold method to segment these images which are in non-uniform illumination conditions. In order to solve the problem, this paper proposed an annulus dividing threshold method based on the classical threshold method. The experiment demonstrated the proposed algorithm outperforms the other traditional methods.
\end{abstract}

Keywords: Non-uniform illumination, Image processing.

\section{Introduction}

With the development of computer technology, the digital image processing has become an important method for particle detection. Its aim is to obtain the accurate appearance and outline information of the particle images, then measure the characteristic parameters of particles and analyze them, that can create conditions for making proper use of them and get better understanding of their properties. However, there is not any segmentation technique can universally applicable, which works equally well for all kinds of images. Therefore, various segmentation approaches have been developed which perform differently for different applications as well for different types of images. In the video supervisory systems, such as underwater detecting, industrial endoscope or pipeline detecting, because of the properties of camera, the impact of camera conditions and the different absorption and reflection properties to light sources on partial surface of detected objects, it results in that the objects have non-uniform exposure to light. These images contain the characteristics of the partial surface are bright and some are dark ${ }^{(1)}$. It influences the precision of the detection and analysis of the results. Non-uniform light field illumination in the image generates background noise which is mixed with signals together can lead to low image contrast or unbalance gray scales and fuzzy edge of objects. It not only destroys the real information of the images, but also seriously impacts the visual effects of the images. Hence in the video supervisory systems it is required to find a fast and effective method which can eliminate the non-uniform illumination on the image. There is lot of research done for the image segmentation with non-uniform illumination ${ }^{(2)}$.

In this paper, first, we use contrast limited adaptive histogram equalization (CLAHE) to enhance the image, after enhancing the image quality, segmentation is performed to extract the desired objects. The remaining of the paper is organized as follows: Next section introduces the various methods for non-uniform correction and discusses the particularities of them; Section 3 describes CHAHE method. Section 4 discusses the segmentation methods and in Section 5 are the results. Finally, the last section gives some conclusion and future work.

\section{Tradition Methods Of Non-uniform Illumination Correction}

A variety of methods have been used for eliminating non-uniform illumination from images. Non-uniform illumination correction technique based on space scope is generally to obtain an estimate of the background image by a variety of computing to the image, and then estimate the original image to eliminate the effects of non-uniform illumination. The following are several methods which are often used in space scope.

\subsection{Histogram Equalization}

In order to enhance the dynamic range of gray value of 
pixels and to improve the overall image contrast effect, histogram equalization is used frequently. Its basic idea is to transform the original image histogram into the form with uniform distribution.

Set up an image with $n$ pixels, which are divided into $L$ gray-scales and $n_{k}$ represent the frequency $r_{k}$ of the $k$ gray-scale, and then the probability of the $k$ gray-level are as follows:

$$
p_{r}\left(r_{k}\right)=\frac{n_{k}}{n} \quad\left(0 \leq r_{k} \leq 1, k=0,1, \cdots L-1\right)
$$

Then the transfer function can be expressed as follows:

$$
s_{k}=T_{\left(r_{k}\right)}=\sum_{j=0}^{k} p_{r}\left(r_{j}\right)=\sum_{j=0}^{k} \frac{n_{j}}{n}
$$

By the above method, many intensive part of the gray scale in histogram distribution has been stretched and the thin part is compressed, get a result of achieving the image contrast enhancement. But, histogram equalization method cannot consider location information of the pixels; it can only consider statistical information of the gray level. So, after the transforming, the gray-scales of the image are seduced, some details are also disappeared. Only with this method, a better result cannot be obtained ${ }^{(3)}$.

\subsection{Sorting correction}

If the background of any specific area in the image is always brighter or always darker than the target, we will check the brightness values of the neighborhood of the current pixel, and then replace the pixel brightness with the lowest brightness. This operation is known as the minimum filtering in sort statistics filtering. Repeat this process until the target of the image is disappeared. The background image is the rest. By subtracting the background image from the original image, certain degree of light-filed correction can be achieved $^{(4)}$. However, this method is very slow. In order to achieve the desired effect, smooth handling is needed for complex background image, which is more cumbersome.

\subsection{Background Fitting}

With human-computer interaction or automatic methods, some researchers select some points of the background image, and these points will be fitted into the dual coordinate function by using the least square method. Then the background image is subtracted from the original image. The main steps are image segmentation, re-sampling and interpolation operations and so on.

Although, in the processing of details in the image, non-uniform illumination correction based on above methods is advisable, but these methods do not consider the frequency characteristics of image formation, and if there is a lot of noise in the image, the result is not good. Hence, in this paper, we proposed image segmentation based on the enhancement method. First, we use a novel contrast limited adaptive histogram equalization enhancement method to enhance the image with the non-uniform illumination, then segment it by using an annulus dividing threshold method based on the local threshold method.

\section{Contrast Limited Adaptive Histogram Equalization}

Contrast limited Adaptive Histogram Equalization (CLAHE), is an improved version of AHE (Adaptive Histogram Equalization).The CLAHE algorithm partitions the images into contextual regions and each one. Overcome the limitations of standard histogram equalization. It divides the images into contextual regions and applies the histogram equalization to each one. A variety of adaptive CLAHE techniques are provided. Sharp field edges can be maintained by selective enhancement within the field boundaries. Noise can be reduced while maintaining the high spatial frequency content of the image by applying a combination of CLAHE, median filtration and edge sharpening. The expression of modified gray levels for standard CHAHE method with uniform distribution can be given as:

$$
g=\left[g_{\text {max }}-g_{\text {min }}\right] * p(f)+g_{\text {min }}
$$

where $g$ is the computed pixel value, $g_{\max }$ is the maximum pixel value; $g_{\min }$ is the minimum pixel value. For exponential distribution gray level can be adapted as:

$$
g=g_{\text {min }}-\left(\frac{1}{\alpha}\right) * \ln [1-p(f)]
$$

where $\alpha$ is a clip parameter. CLAHE method operates on tiles which is one small region in the image, rather than the whole image. Each tile contrast is enhanced, so the histogram of the output region approximately matches the histogram $^{(5)}$.

\section{Annulus Division}

There are two important parameters for annulus division; one is determining the center coordinate $c_{0}$, the other is $d_{0}$ the width of annulus. $c_{o}$ is the focus of the image bright areas which can be understood as the nearest pixel in the plane from the camera . $d_{0}$ is determined by two factors, 
one is the size of target, the other is the attenuation of brightness. The center of gravity in image bright areas is the focus of annulus.

Calculate the average gray value of enhanced image, the average gray value of Figure 1 (a) is 52.8, and use the average gray value as a threshold binary image (As Figure 1(b)). Then as following formula, calculate the value of the center of gravity in white area, the value is $c_{0}(x, y)$

$$
\left\{\begin{array}{l}
x=\frac{1}{n} \sum_{i=1}^{n} x_{i} \\
y=\frac{1}{n} \sum_{j=1}^{n} y_{j}
\end{array}\right.
$$

where $n$ is the number of white pixels, $\left(x_{i}, y_{j}\right)$ the value of white pixel.

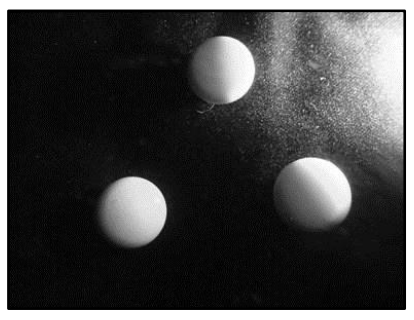

(a)

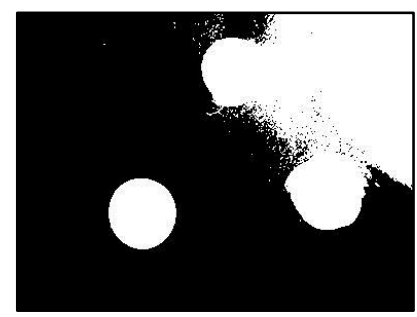

(b)
Figure 1 Determination of the center $c_{0}$ (a) Gray image

(b) The binarized image

Two factors need to be considered to determine the $d_{0}$. One is the size of target; the other is the attenuation of brightness. Through a large number of simulation experiments, the result is more satisfactory if the value of $d_{0}$ is between $0.6-1.0$.

First, the image is divided into four quadrants as $c_{0}$ is the origin point; According to the value of $d_{0}$, each quadrant is divided into a number of annulus, as shown in Figure 2. According to the formula (6) and (7), we can determine the quadrant of pixel $(x, y)$. As shown in Figure 2.

$$
\begin{aligned}
& \{\Delta x, \Delta y\}=\left\{x-x_{0}, y-y_{0}\right\} \\
& \left(\sqrt{\left(x-x_{0}\right)^{2}+\left(y-y_{0}\right)^{2}} \% d\right)
\end{aligned}
$$

where, "\%" is the remainder operator. The symbol of $\Delta x, \Delta y$ determines position of the quadrants.

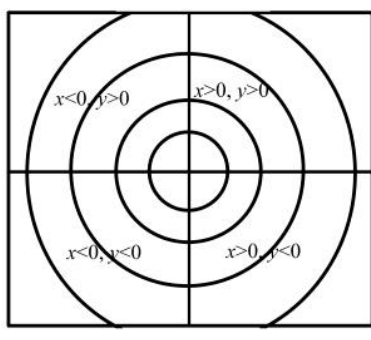

(a)

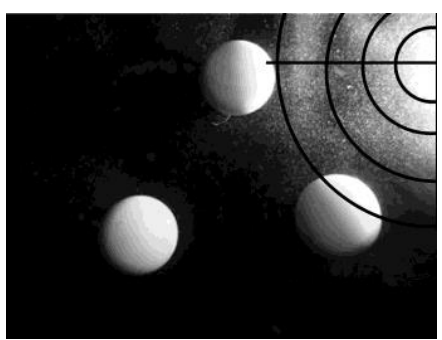

(b)
Figure 2 Schematic diagram of sub-images with annulus division (a) Annulus division (b) Division of sub-images

We use OTSU method to find the optimal threshold of sub-images ${ }^{(6)}$, OTSU is described as follows:

$$
\begin{aligned}
& u=\omega_{0} * u_{0}+\omega_{1} * u_{1} \\
& g=\omega_{0}\left(u_{0}-u\right)^{2}+\omega_{1}\left(u_{1}-u\right)^{2} \\
& g=\omega_{0} \omega_{1}\left(u_{0}-u_{1}\right)^{2}
\end{aligned}
$$

where $u$ is the total average gray value of sub images; $\omega_{0}$ is the ratio of target pixels and pixels in sub images; $u_{0}$ is the average gray value of target pixels; $\omega_{1}$ is the ration of background pixels and pixels in sub images; $u_{l}$ is the average gray value of background pixels; formula (10) is the Equivalence formula of formula(9). The threshold of sub image is $T$ only when $T$ makes formula (10) get maximum value, $T$ is optimal threshold. Then we segment the image with threshold $T$.

\section{Experiments}

To estimate the effectiveness of our proposed method, we apply it to a button image. Figure 3(a) is the input image. Firstly, contrast limited adaptive histogram equalization (CLAHE) is to be used to enhance the image, after enhancing the image quality, we use annulus division to remove the non-uniform illumination in the image, as shown in Figure 3(b); Next, Otsu method is to be used for segmenting the image, the result is shown as in Figure 3(c). 


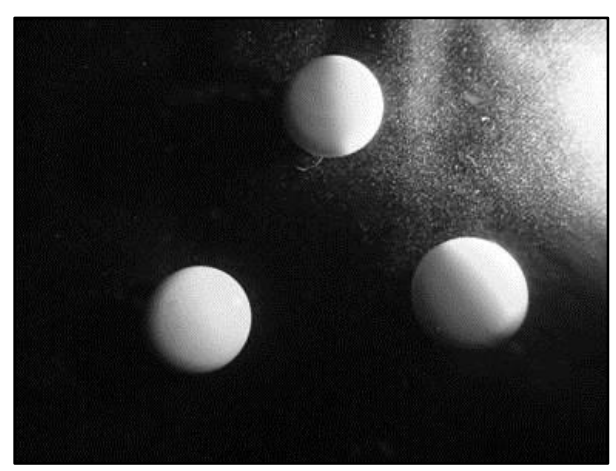

(a)

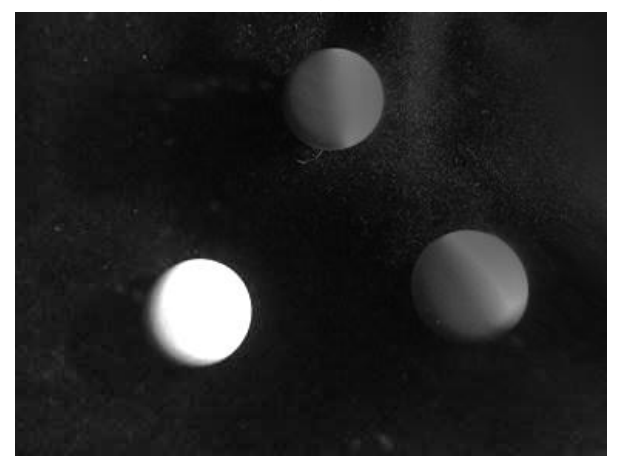

(b)

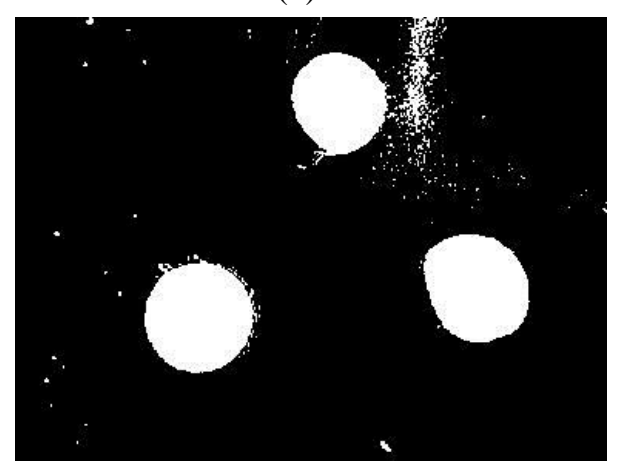

(c)

Figure 3 Experiment Results (a) input image (b) image with removing non-uniform illumination (c) image of segmentation

Through the above experiment, we can find that the processed method can detect the buttons well. However, there also contains some noisy edges, because of the drawbacks of the automatic Otsu segmentation.

\section{Conclusion}

In this paper, we have presented an annulus optimal threshold method based for non-uniform illumination image correction. Through experiments, for image with non-uniform illumination, the proposed method can get better result. In future, we will adapt a much effective methods for solving the non-uniform illumination problem.

\section{Acknowledgment}

This work is supported by Grant in Aid for Japan Society for the Promotion of Science Research Follows (No.25-10713) and Grant in Aid for Non-Japanese Researchers of NEC C\&C Foundation. The authors are grateful to the anonymous referee for a careful checking of the details and for helpful comments that improved this paper.

\section{References}

(1) J. Zheng, C.G. Xu, D.G. Xiao: The Technique of Digit Image's Illumination Uneven Elimination. Transactions of Beijing Institute of Technology, 2003, 23(3):pp285-289.

(2) Z.Y. Lu, X.P. Yan: The Pretreatment of Segmentation on Distributing Uneven Background Brightness of Wear Particle Image. Lubrication Engineering, 2005, (5):pp34-37.

(3) M. Abdullah-Al-Wadud, Md. H. Kabir, M. A. Akber Dewan, O. Chae: A dynamic histogram equalization for image contrast enhancement, IEEE Transactions. Consumer Electron, 2007, pp593- 600.

(4) S.P. Zhang, C.T. Zhang: Sea Surface Image Enhancement Based on Homomorphic Filtering, Ocean Technology,2007, 26(1):pp6-9.

(5) K. Zuiderveld: Contrast Limited Adaptive Histogram Equalization. Graphics Gems IV, 1994, pp. 474-485.

(6) J. Zhang, J.L. Hu: Image segmentation based on 2D Otsu method with histogram analysis. International Conference on Computer Science and Software Engineering, 2008, pp105-108. 